\title{
A Case of Wolfram Syndrome Presenting with Restlessness
}

\author{
SGM MOWLA ${ }^{\mathrm{a}}$, MT MIAH ${ }^{\mathrm{b}}$, ARAHMAN $^{\mathrm{c}}$, SS SHONCHOI ${ }^{\mathrm{d}}$, MN ALAM $^{\mathrm{e}}$, M SALIM $^{\mathrm{f}}$
}

\begin{abstract}
Summary:
Wolfram Syndrome (DIDMOAD) is a rare genetic disorder presenting with Diabetes Insipidus, Diabetes Mellitus, Optic Atrophy, Deafness and some other neurological, reproductive, hormonal, urological and psychic problems. About 200 cases have been reported so far. Here we present a 25 years old Bangladeshi male having early onset Diabetes Mellitus, optic atrophy, deafness and many other features consistent with Wolfram Syndrome. We examined the patient thoroughly and did necessary
\end{abstract}

\section{Introduction:}

Wolfram Syndrome is a rare neuro-degenerative disorder involving central nervous system, peripheral nerves and neuro-endocrine tissue. This rare genetic disorder, also known as DIDMOAD (Diabetes Insipidus, Diabetes Mellitus, Optic Atrophy, Deafness) presents with early onset diabetes mellitus and optic atrophy. Patients are most likely to develop diabetes insipidus, deafness , urinary tract and neurological abnormalities. ${ }^{1}$ It is an autosomal recessive disorder and the causative gene maps to chromosome $4 \mathrm{p} 16.1$. The mutation causes loss of function of the protein, wolframin that has important role in the homeostasis of endoplasmic reticulum. Recently, another causative gene, CISD2, has been identified in patients with a type of Wolfram Syndrome (WFS2) resulting in early optic atrophy, DM, deafness, decreased life span but no diabetes insipidus ${ }^{2}$. Treatment is mostly supportive. Prognosis is very poor with the highest life expectancy of 35 years in $60 \%$ of the patients ${ }^{3}$. This case report describes a 25 years old male of Wolfram Syndrome with an affected sibling.

a. Dr. Syed Ghulam Mogni Mowla, Assistant Professor, Department of Medicine , Dhaka Medical College.

b. Dr. Md. Titu Miah, Associate Professor, Department of Medicine, Dhaka Medical College.

c. Dr. Ashraf-ur-Rahman, Assistant Registrar, Department of Medicine, Dhaka Medical College Hospital.

d. Dr. Shamsuddoha Sarker Shonchoi, Medical Officer, Department of Medicine, Dhaka Medical College Hospital.

e. Dr. Muhammad Nazmul Alam,, Medical Officer, Dept. of Medicine, Dhaka Medical College Hospital.

f. Dr. Mohammad Salim, Medical Officer, Dept. of Medicine, Dhaka Medical College Hospital.

Address of Correspondence: Dr. Syed Ghulam Mogni Mowla, Assistant Professor , Department of Medicine, Dhaka Medical College, Mobile: 01534307198, Email: dr_mogni@yahoo.com Received: 3 June, 2014

Accepted: 21 October, 2015 investigations to confirm our diagnosis. As there is no cure of this disorder, we gave symptomatic and supportive treatment to the patient to make his life easier. Although the outcome is unrewarding, such patients will be kept in regular follow up for early detection of new complications and possible solutions.

Keywords: Wolfram Syndrome, Diabetes insipidus, Diabetes mellitus, optic atrophy.

(J Banagladesh Coll Phys Surg 2016; 34: 42-44)

\section{Background:}

Wolfram Syndrome was named after a physician D J Wolfram who reported 4 cases in $1938^{4}$. Frequency is 1 in 100000 to 1 in $7000008^{3}$. Parental consanguinity is often present in affected siblings. This rare complex hereditary disorder manifests as Young onset non immune insulin dependent diabetes mellitus and progressive optic atrophy in all patients. In $70 \%$ patient diabetes incipidus and sensory neural deafness is added where it is referred to as DIDMOAD ${ }^{3}$. The natural history of Wolfram syndrome was described in 45 individuals from 29 families in $U K^{5}$. By the age of 20 years, $64 \%$ had hearing impairment and $60 \%$ of all (mean age 16 years, range $5-32$ years) had one or more of the following: cerebellar ataxia, peripheral neuropathy, intellectual disability, dementia, psychiatric illness and urinary tract atony. Constipation, chronic diarrhea and other bowel dysfunction is reported in $25 \%$ cases $^{6}$.

\section{Case Report:}

Our case is a 25 years old Asian male from Tongipara, Munshigonj, born to consanguineous parents, presented with restlessness for 1 day, and gradual loss of vision for 7 years. He was a diagnosed case of diabetes mellitus and was on insulin for 18 years. He also had ployuria , polydypsia, occasional episodes of altered consciousness and poor control of diabetes mellitus. The patient developed hearing difficulty for 3 years and his intellectual function was below average.

The patient has one affected sister of 16 years having diabetes mellitus for 5 years and dimness of vision for 1.5 years. She also has hearing difficulty.

Examination revealed a height of 56.5 inches, weight 45 kg, BMI 21.51, decreased axillary hair, absence of chest hair, normal pubic hair and presence of gynaecomastia. 
Cranial nerve examination showed- decreased smell perception, visual aquity decreased to perception of light, fundoscopy revealed- pseudophakia of both eyes, liquefied vitreous, bilateral primary optic atrophy and micro aneurysm. Sensory neural hearing impairment was noted. Testicular atrophy with soft testes was found.

The patient underwent some investigations. CBC showed Hb \%-9.1 gm/dl , WBC-20.9×103/cmm, RBC$3.82 \times 10^{`} 6$ /cmm ,DC- N-91.1 \%, Hct- 29.7\%. Urine R/E showed Albumin: +,Pus cell:5-10/ HPF, Epithelial cells: 4-6 / HPF, RBC: 2-4 / HPF , presence of granular casts. Serum sodium was high ( $150 \mathrm{mmol} / \mathrm{L}$ ) initially but later it became normal. USG of whole abdomen revealed cholelithiasis, bilateral hydronephrosis and cystitis. Audiogram showed - bilateral moderate sensory neural hearing loss. Some hormonal studies were done with the following results- Follicle Stimulating Hormone(FSH): 37.06 mIU/ml (Very High), Leutinizing Hormone (LH): 12.49mIU/ml (Slightly Raised), Testosterone: $0.40 \mathrm{nmol} / \mathrm{L}$ (Very low, Normally 10.4$35.71 \mathrm{nmol} / \mathrm{L}$ for 20 -30 years male). Plasma Osmolality: 282, Urinary Osmolality: 283 , urinary specific gravity was 1.008. MRI of brain was normal.

\section{Discussion:}

Wolfram syndrome usually presents with non autoimmune, non HLA linked diabetes mellitus with optic atrophy in $1^{\text {st }}$ decade, diabetes incipidus and sensory neural deafness in $2^{\text {nd }}$ decade, renal tract abnormalities in the $3^{\text {rd }}$ decade and multiple neurological abnormalities like cerebellar ataxia, myoclonus and psychiatric illness early in the $4^{\text {th }}$ decade ${ }^{5}$. In our case, the patient was first diagnosed as having IDDM at the age of 7 years that is in the $1^{\text {st }}$ decade and developed gradual dimness of vision for 7 years that is approximately at the age of 18 years. He had no evidence of diabetic retinopathy. And for the last 3 years he can only differentiate light from darkness.

The patient had polyurea and urinary osmolality was slightly decreased. Water deprivation test could not be done as the patient and relatives refused after explaining the procedure. So the presence of diabetes incipidus could not be proven.

Our patient developed hearing difficulty around the age of 22 years. It was also proven by audiogram. Audiograms show a down sloping progressive pattern of hearing loss in $\mathrm{WS}^{7}$.
A wide range of urological abnormalities are seen in patients with Wolfram Syndrome including varying degrees of upper urinary tract dilatations, recurrent bladder infection, and bladder dysfunction ${ }^{4}$. USG of our patient revealed bilateral hydronephrosis and cystitis.

The presence of decreased hair, gynaecomastia, testicular atrophy and high gonadotropin levels proves gonadal failure in our patient. Hypogonadism in Wolfram syndrome is more common in males, while females usually retain their ability to become pregnant ${ }^{8}$. A few episodes of aggressive behavior was noted in our patient.

Our patient has an affected sister and his parents are $1^{\text {st }}$ cousins. This type of family history is very common in patients of Wolfram Syndrome.

There is no definitive treatment of these patients. Supportive care like insulin for DM, vasopressin for DI, hearing aids and hormone replacements along with prevention of secondary complications like those of DM and regular surveillance to detect manifestations early - are the principles of management. Genetic counseling must be provided that at conception, each sib of an affected individual has a $25 \%$ chance of being affected, a $50 \%$ chance of being an asymptyomatic carrier and a $25 \%$ chance to be unaffected. The prognosis is very poor with a premature death before the age of 35 years in $60 \%$ patients $^{3}$. Common causes of death are hypoglycemic coma, status epilepticus, ESRD and suicide $^{8}$.

\section{Conclusion:}

Wolfram Syndrome is a rare genetic disorder but whenever a young patient presents with early onset diabetes mellitus and optic atrophy, the possibility of Wolfram Syndrome should always be kept in mind. Subtle symptoms such as attitude change, growing reading difficulties in the history of children or adolescents with antibody negative and ketone negative DM should alert the treating physician for further evaluation ${ }^{9}$. Although the outcome is unrewarding, steps should be taken to improve the quality of life and to prevent complications. Multidisciplinary approach should be instituted as early as possible to make the patient's short span of life worthwhile. 


\section{Consent:}

Written informed consent was obtained from the patient's father for publication of the case report and accompanying images. A copy of the written consent is available for review by the Editor in Chief of this journal.

\section{Conflict of Interest:}

The authors declare that they have no conflict interests.

\section{References:}

1. Concetta Aloi, Alessandro Salina, Lorenzo Pasquali, Francesca Lugani , Katia Perri, Chiara Russo, Ramona Tallone, Gian Marco Ghiggeri, Renata Lorini, Giuseppe d’Annunzio. Wolfram Syndrome: New Mutations, Different Phenotype. PLoS One. 2012 Jan ; 7(1): e29150.(Available from Net library)

2. Rigoli L, Di Bella C. Wolfram syndrome 1 and Wolfram syndrome 2. Curr Opin Pediatr. 2012 Aug; 24(4):512-7

3. Viswanathan V, Medempudi S, Kadiri M. Wolfram Syndrome. J Assoc Physicians India. 2008 Mar;56:197-9.
4. Masoud Reza Manaviat, Maryam Rashidi, Seyed Mohammad Mohammadi .Wolfram Syndrome presenting with optic atrophy and diabetes mellitus: two case reports. Cases Journal . 2009; 2:9355

5. Barrett TG, Bundey SE, Macleod AF. Neurodegeneration and diabetes: UK nationwide study of Wolfram (DIDMOAD) syndrome. Lancet; 1995; 346(8988): 1458-1463.

6. Liu Z, Sakakibara R, Uchiyam T, et al. Bowel dysfunction in Wolfram Syndrome. Diabetes Care. 2006; 29:472-3.

7. Pennings RJ, Huygen PL, van den Ouweland JM, et al. Sex-related hearing impairment in Wolfram syndrome patients identified inactivating WFS1 mutations. Audiol Neurootol. 2004;9:51-62

8. Tranebjaerg L, Barrett T, Rendtorff ND. WFS1 related disorders. 2009 Feb 24 [updated 2013 dec 19]. In: Pagon RA, Adam MP, Arbinger $\mathrm{HH}$, et al; editors. GeneReviews [internet]. Available from: http://www.nlm.nih.gov/books/ NBK4144/

9. Boettcher C, Brosig B, Zimmer KP, Wudy SA. Journal of Pediatric Endocrinology and Metabolism. 2011 Mar; 24(1-2):71-74. 